\title{
Recurrent Sweet's Syndrome in a Case of Smoldering Multiple Myeloma and Chronic Hepatitis C
}

\section{Mark Obri ${ }^{1,2 *}$, Arjun Pandya ${ }^{1,2}$, James Lerakis ${ }^{1,2}$, Alexander Isla ${ }^{1,2}$, Michael Rich MD $^{2}$, Ania Henning MD $^{2}$}

${ }^{1}$ Northeastern Ohio Medical University, Rootstown, Ohio, USA

${ }^{2}$ Summa Health System, Akron, Ohio, USA

*Corresponding Author: Mark Obri, Northeastern Ohio Medical University, Rootstown and Summa Health System, Akron, Ohio, USA.
Received: April 26, 2020

Published: June 09, 2020

(C) All rights are reserved by Mark Obri., et al.

\begin{abstract}
Background: Sweet's Syndrome, also known as acute febrile neutrophilic dermatosis, is a rare inflammatory skin disorder that presents as a rapid onset of tender, inflammatory lesions, which usually appear on the arms, face, and neck. It is histologically characterized by a neutrophilic infiltrate in the dermis with karyorrhexis and massive papillary dermal edema. Sweet's is subdivided into three classifications based on etiology: classical, malignancy-associated, and drug-induced.

Case Presentation: We present the case of a 55 year old African American woman with a past medical history of smoldering multiple myeloma and hepatitis $\mathrm{C}$ who presented to the emergency department with widespread tender bullous lesions on the extremities, trunk, inguinal area, and vulva, along with accompanying polyarthritis. The patient was treated empirically with intravenous methylprednisolone for suspected autoimmune disease following rule out of infectious etiology and vasculitis. Biopsies of the lesions on subsequent hospitalization demonstrated neutrophilic infiltrate of the dermis, and the patient was diagnosed with Sweet's Syndrome.

Conclusion: It is unknown whether this patient's Sweet's Syndrome was secondary to her smoldering multiple myeloma, hepatitis C, or idiopathic. We believe that smoldering multiple myeloma was the likely underlying culprit given the link between blood malignancies and Sweet's Syndrome, the widespread presentation of the lesions, and similar presentations reported in cases of multiple myeloma. Based on our case study, we recommend patients diagnosed with Sweet's Syndrome receive appropriate workup for blood malignancies and hepatitis C.

Keywords: Sweet's Syndrome; Smoldering Multiple Myeloma; Hepatitis C
\end{abstract}

\section{Introduction}

Sweet's Syndrome, also known as acute febrile neutrophilic dermatosis, is a rare dermatological disorder that is characterized by rapid onset of extremely tender and erythematous nodules, plaques, or bullous lesions, most often appearing on the extremities. This disorder can also be accompanied by fever, myalgia, polyarthralgia, and neutrophilia. Diagnosis is made with biopsy demonstrating neutrophilic infiltrate in the upper dermis. The first case of Sweet's Syndrome was published in the 1964 British Journal of Dermatology by Dr. Robert Douglas Sweet, who had documented eight female patients with this disease over a fifteen-year period [13]. Contemporarily, patients diagnosed with Sweet's Syndrome must fulfill two of two major criteria and two of four minor criteria (Table 1) [14]. Sweet's Syndrome is then subcategorized as classical, drug-induced, or malignancy-associated based on suspected etiology. Classical Sweet's Syndrome encompasses idiopathic cases and those with etiologies aside from cancer, most commonly pregnancy, recent upper respiratory infection, or recent gastrointestinal infection [4]. Drug therapies linked with Sweet's include G-CSF and all-trans-retinoic acid [11]. Malignancy-associated cases most often involve hematolymphoid malignancies such as leukemias, and there have been cases of Sweet's Syndrome reported in the setting of multiple myeloma; however, to our knowledge no cases of Sweet's Syndrome have been reported in the setting of smoldering multiple myeloma. Sweet's Syndrome may also occur in association hepatitis $\mathrm{C}$, a virus which involves secondary dermatologic manifestations in more than $45 \%$ of cases. Here we report a case of atypically presenting Sweet's Syndrome in a patient whose medical history includes multiple plausible causes, obscuring deduction of the exact etiology and precise subcategorization. 


\begin{tabular}{|c|c|}
\hline Major Criteria & Minor Criteria \\
\hline $\begin{array}{l}\text { - Abrupt onset of } \\
\text { painful erythema- } \\
\text { tous plaques or nod- } \\
\text { ules } \\
\text { - Histopathologic } \\
\text { evidence of a dense } \\
\text { neutrophilic infil- } \\
\text { trate without evi- } \\
\text { dence of leukocyto- } \\
\text { clastic vasculitis }\end{array}$ & 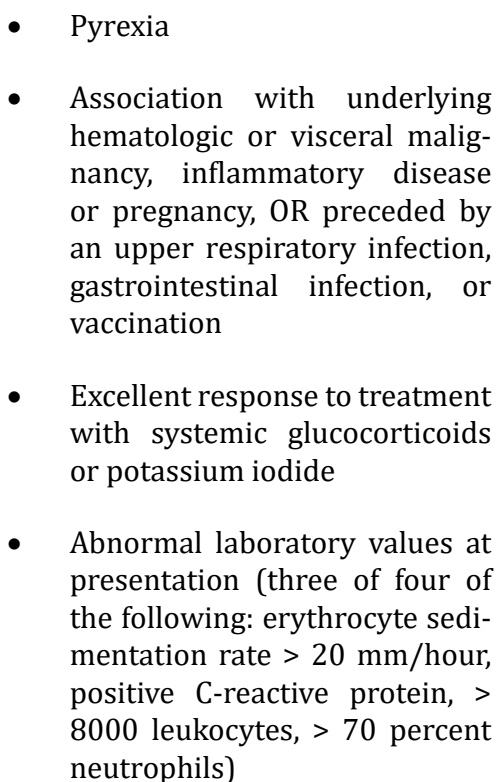 \\
\hline
\end{tabular}

\section{Table 1}

\section{Case Report}

This patient is a 55 year old African American woman with a past medical history of untreated smoldering multiple myeloma, untreated chronic hepatitis $\mathrm{C}$, seizure disorder, hypertension, and an episode of acute pharyngitis two weeks prior, who presented to the emergency department with a two day history of sudden onset painful bullous lesions on her arms, legs, trunk, and genital area. The patient endorsed fevers, chills, nausea, arthralgia/myalgia, and vomiting that began with the eruptions two days prior to admission. The lesions were present in different stages, with some having ruptured to release serous or sanguineous drainage. The patient's vital signs showed a blood pressure of 149/85, pulse 121, temperature $98.8^{\circ} \mathrm{F}$, and respiratory rate of 18 . Despite her stable condition, the patient was in obvious acute distress. The patient had presented three months prior with similar lesions, at which time she underwent extensive infectious disease and autoimmune workup. That workup, which did not include skin biopsy, failed to establish a diagnosis, and the patient was treated empirically with intravenous methylprednisolone for suspicion of an undetermined autoimmune etiology. The treatment resulted in dramatic improvement of the lesions. At the time of her second presentation, pertinent blood work included neutrophilia and an IgG level of $3029.9 \mathrm{mg} / \mathrm{dL}$. Her hepatitis C RNA viral load 7 years ago was 350,000 copies/mL. A punch biopsy of a lesion on her left lateral thigh demonstrated a diffuse neutrophilic infiltrate with scattered karyorrhectic debris and extravasated red blood cells in the dermis. These biopsy findings confirmed the diagnosis of Sweet's Syndrome (See additional results).

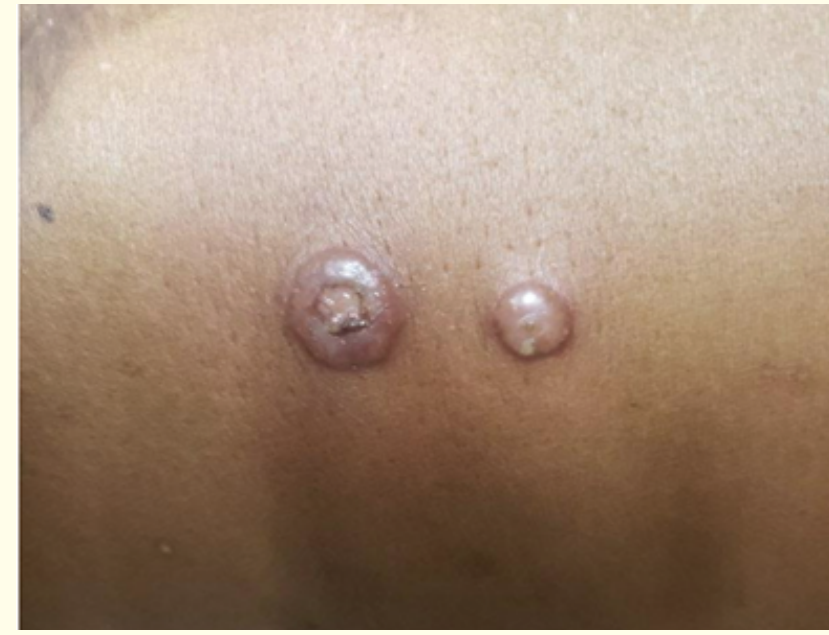

Figure 1

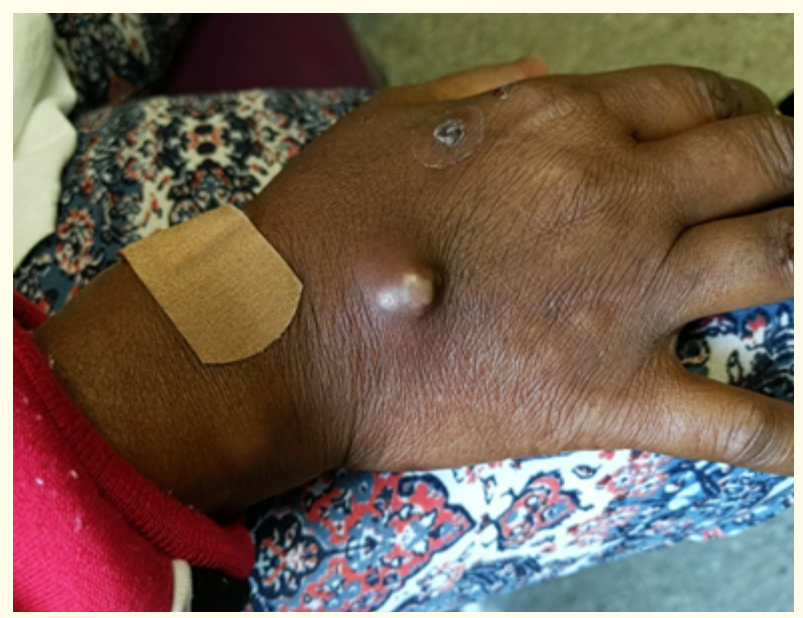

Figure 2

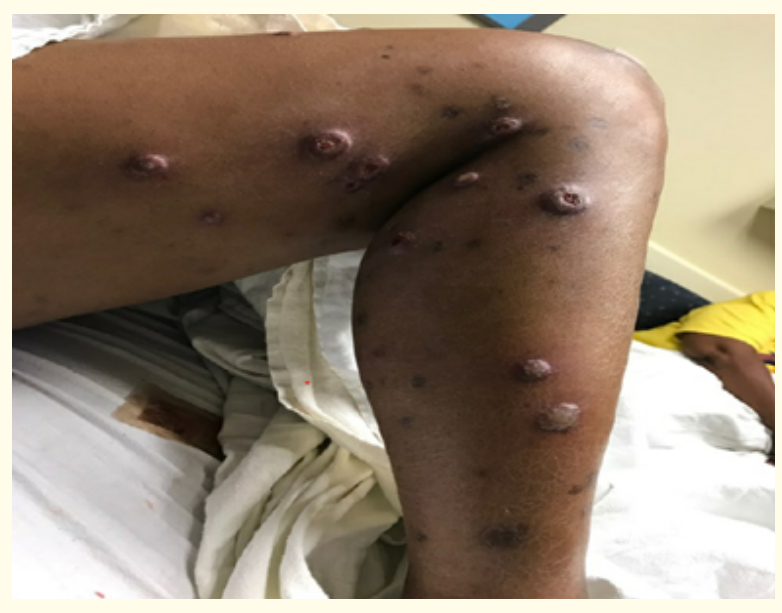

Figure 3 


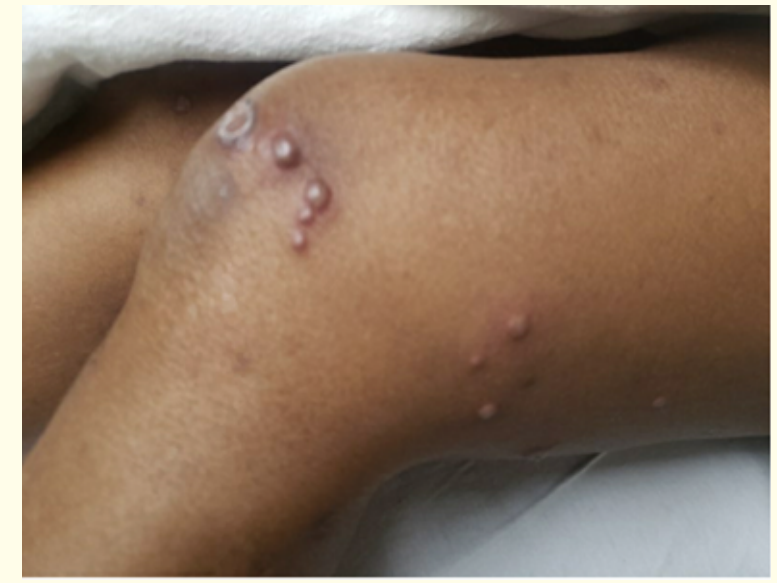

Figure 4

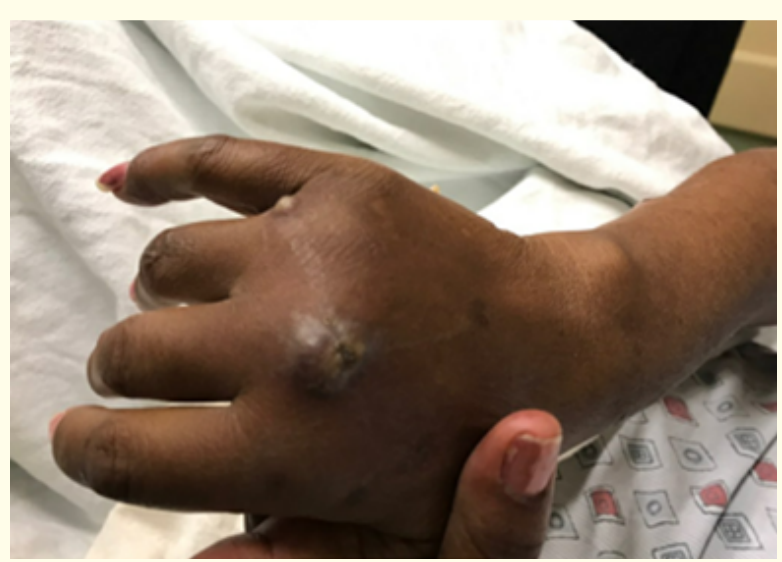

Figure 5

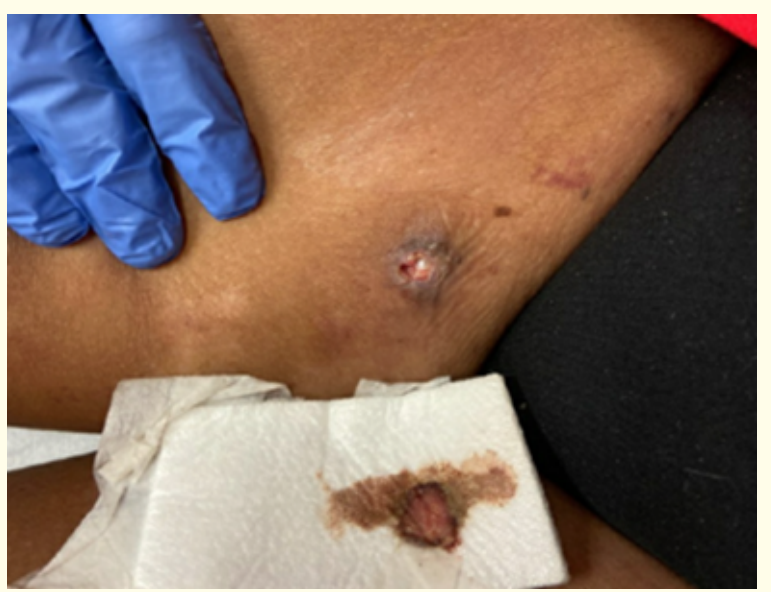

Figure 6

Figure 1-6: Representing bullous lesions on the patients trunk and extremities.

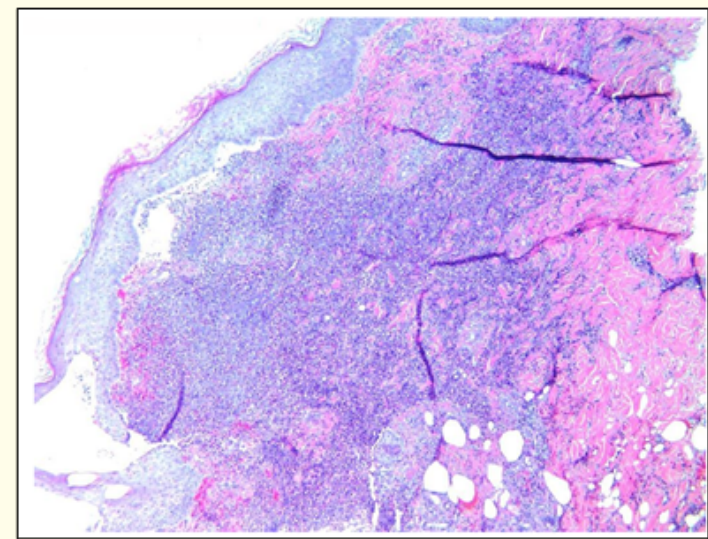

Biopsy 1: Low power view of a diffuse dermal inflammatory infiltrate consisting predominantly of neutrophils.

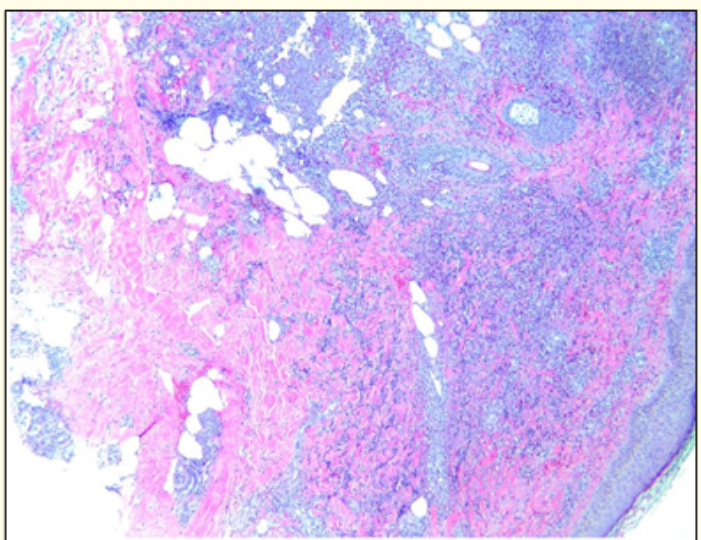

Biopsy 2: Dermis with diffuse dermal infiltrate composed of neutrophils, karyorrhectic debris, and extravasated red blood cells.

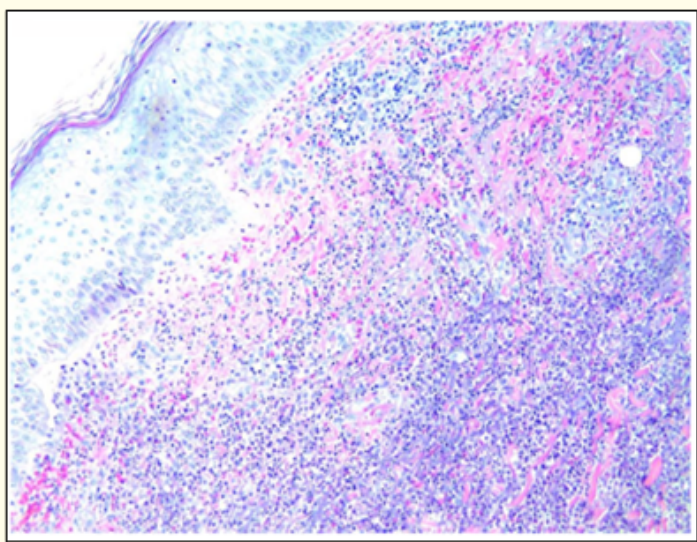

Biopsy 3: Periadnexal and perivascular inflammatory infiltrate composed mostly of neutrophils as well as scattered eosinophils, lymphocytes and histiocytes (Biopsy pictures and captions courtesy of Ania Henning MD). 
The patient's condition improved once again following intravenous administration of methylprednisolone. She was discharged with an oral prednisone taper. Patient showed improvement in the follow up appointment one week following discharge.

\section{Discussion}

This patient exhibits a case of recurrent Sweet's Syndrome possibly secondary to acute pharyngitis, smoldering multiple myeloma, or chronic hepatitis C. The patient did have an episode of pharyngitis two weeks prior to her second eruption; however, the recurrent nature of the lesions appears to indicate that the underlying etiology is itself chronic. As such, we believe that the patient's multiple presentations could be a precipitant of her smoldering multiple myeloma or her chronic hepatitis C, particularly considering that both disorders remain untreated. The patient was diagnosed with both underlying conditions prior to the onset of these lesions.

Multiple previous cases of Sweet's Syndrome in the setting of hepatitis $\mathrm{C}$ have reported dermatological manifestations localized only to the dorsum of the hands, whereas our patient exhibits Systemic Sweet's lesions involving the trunk, genitals, and all extremities in addition to the dorsum of the hands [5]. Such signs diminish the likelihood that hepatitis $\mathrm{C}$ is the underlying cause.

Although the exact pathophysiological association between blood malignancies and Sweet's Syndrome is currently unknown, about $11 \%$ of cases of Sweet's Syndrome are associated with either a blood malignancy or a solid tumor [3]. Cases of recurrent Sweet's Syndrome have been reported in the setting of multiple myeloma, with patients demonstrating disseminated lesions on the trunk and extremities. Our patient's manifestation is similar with the rare addition of genital involvement. Furthermore, our patient has not yet been diagnosed with multiple myeloma; hematology and oncology has rendered a diagnosis of smoldering myeloma owing to the patient's relatively normal blood counts, calcium levels, and kidney function. Moreover, the patient's IgG levels of 3029.9 $\mathrm{mg} / \mathrm{dL}$, while well above normal, are not within the typical $\mathrm{IgG}$ range for multiple myeloma, which is in excess of $10000 \mathrm{mg} / \mathrm{dL}$. To our knowledge, there have been no reported cases of Sweet's Syndrome in the setting of smoldering multiple myeloma. Even so, in light of our patient's presentation bearing more resemblance to the presentations previously reported in association with multiple myeloma coupled with evidence of recurrence, we believe the untreated blood malignancy smoldering multiple myeloma to be the most likely underlying cause.

Sweet's syndrome has had a previously documented association with multiple myeloma in many settings. Previous case re- ports have seen lesions erupt on solely the dorsum of the hands, to asymmetrically disseminated throughout the body. There has also been a reported case of multiple myeloma causing recurrence of Sweet's Syndrome [8]. It is hypothesized to be a rare paraneoplastic syndrome of multiple myeloma, potentially caused by neutrophil activation secondary to an increase in cytokines. Specifically, Granulocyte-Colony Stimulating Factor, G-CSF, has been hypothesized to be one of the chemical messengers playing a factor in this disease. G-CSF contributes to activation, increased production and chemotaxis of neutrophils. It has been found to be elevated in Sweet's Syndrome, and furthermore has been found to increase IgG FC receptors on neutrophils. This may lead to neutrophil activation and peripheral infiltration into the dermis. Thus, elevated IgG coupled with elevated G-CSF would lead our patient susceptible to Sweet's Syndrome [3].

While the patient did respond to corticosteroid therapy, the first-line treatment for Sweet's, treatment of the underlying cause offers the best chance of preventing further recurrences. Therefore, the patient has initiated treatment of her chronic hepatitis $\mathrm{C}$ and has been referred to hematology-oncology for management of her smoldering multiple myeloma.

\section{Conclusion}

In sum, there are various etiologies of Sweet's Syndrome, and each etiology must be thoroughly explored before a case is ruled idiopathic. We recommend that the workup of Sweet's Syndrome include ruling out multiple myeloma and other associated blood malignancies, as well as hepatitis C. Systemic glucocorticoids have been an effective treatment for this disease.

\section{Additional Resources}

Labs: Lab studies CBC- $\mathrm{Hb}$ of 11.4 and MCV of $108.4 \mathrm{CMP}^{-\mathrm{CO}_{2} \text { of } 19}$ anion gap of 13 and $\mathrm{Cl}$ of 113 . LFT AST of 64

\section{Biopsy Report}

A. Skin, left lateral thigh "vesicle", punch biopsy - diffuse dermal, neutrophilic infiltrate

Comment: The histologic differential includes cellulitis. There is no evidence of a granulomatous component arguing against a deep fungal or atypical mycobacterial infection. Ancillary stains for those organisms will be performed and reported as an addendum. There is no evidence of viral cytopathic changes. If the tissue cultures are negative and if it is determined to be a non-infectious etiology, the differential includes the neutrophilic dermatoses including Sweets Syndrome, rheumatoid neutrophilic dermatosis, and Behcet's disease, amongst others. Extensive deeper sections were reviewed and a vasculitis is not identified. The infiltrate is diffuse and not confined to the sweat glands arguing against eccrine hidradenitis. 
Microscopic description: Sections demonstrate a slightly acanthotic and reactive epidermis. Within the dermis is a diffuse, neutrophilic infiltrate with scattered karyorrhectic debris and extravasated red blood cells. The associated superficial and deep superficial vascular plexus demonstrate endothelial cell swelling but no evidence of fibrinoid necrosis of the blood vessel walls. Scattered eosinophils, lymphocytes, and histiocytes are noted.

B. Skin, left lateral thigh, punch biopsy (DIF) - Negative direct, immunofluorescence

Microscopic description: Direct antibody localization demonstrates no evidence of immunoreactivity for immunoglobulins IgG, IgM, IgA, complement C3, or fibrinogen on sections of frozen skin.

\section{Acknowledgements}

Ania Henning, MD for provision and interpretation of all histological slides included herein.

\section{Bibliography}

1. American Cancer Society. "Tests to Find Multiple Myeloma" (2019).

2. Bayer-Garner IB., et al. "Sweet syndrome in multiple myeloma: a series of six cases". Journal of Cutaneous Pathology 30.4 (2003): 261-264.

3. Belhadjali H., et al. "Sweet's syndrome associated with multiple myeloma”. Acta Dermato-Venereologica 17.1 (2008): 3133.

4. Cleveland Clinic. "Sweet Syndrome" (2019).

5. Coelho JF., et al. "Neutrophilic dermatosis of the dorsal hands associated with chronic hepatitis C virus infection". Indian Journal of Dermatology, Venereology, and Leprology 74.5 (2008): 478-480.

6. Cohen PR. "Sweets syndrome - a comprehensive review of acute febrile neutrophilic dermatosis". Orphanet Journal of Rare Diseases 2.1 (2007): 34.

7. Fauconnier SA., et al. "Neutrophilic dermatosis of the dorsal hands with multiple myeloma". Joint Bone Spine 83.2 (2016): 227.

8. Gurnari C., et al. "Recurrent Sweets syndrome in a patient with multiple myeloma". Clinical Case Reports 6.10 (2018): 1958-1960.

9. Hallberg P., et al. "Acute pancreatitis following medical abortion: Case report". BMC Womens Health 4.1 (2004): 1.
10. Khalid A. "Sweet's syndrome with multiple myeloma". Journal of Applied Hematology 5.3 (2014): 69-70.

11. Merola MD. "Sweet syndrome (acute febrile neutrophilic dermatosis): Pathogenesis, clinical manifestations, and diagnosis". Post TW, ed. UpToDate. Waltham, MA: UpToDate Inc (2019).

12. NORD. "Sweet Syndrome" (1987).

13. Sweet RB. "An acute febrile neutrophilic dermatosis". British Journal of Dermatology 76 (1964): 349-356.

14. Vadde R and Schmidt FJ. "Immunoglobulins: Reference Range, Interpretation, Collection and Panels" (2013).

\section{Assets from publication with us}

- Prompt Acknowledgement after receiving the article

- Thorough Double blinded peer review

- Rapid Publication

- Issue of Publication Certificate

- High visibility of your Published work

Website: $\underline{w w w . a c t a s c i e n t i f i c . c o m / ~}$

Submit Article: www.actascientific.com/submission.php Email us: editor@actascientific.com

Contact us: +919182824667 